\title{
Synthesis and Properties of Rosin-Based Dispersant for Coal-Water Slurry
}

\author{
Junguo $\mathrm{Li}^{1, \mathrm{a}}$, Guanghua Zhang ${ }^{1, \mathrm{~b}}$, Wenjing $\mathrm{Han}^{1, \mathrm{c}}$ and Junfeng $\mathrm{Zhu}^{1, \mathrm{~d}}$ \\ Key laboratory of Auxiliary Chemistry \& Technology for Chemical Industry, Ministry of Education, \\ Shaanxi University of Science \& Technology, Xi'an 710021, P. R. China \\ aemail: lijunguo@sust.edu.cn; ${ }^{b}$ email: zhanggh@sust.edu.cn;

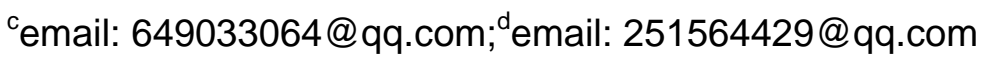

Keywords: Rosin derivative; Coal-water slurry; Dispersant; Dendrimer

\begin{abstract}
The main objective of the present work was to synthesize a new type of rosin-based dispersant for Chinese Shenhua coal-water slurry (CWS) and evaluate it's application performances for CWS preparation. In this study, 1.0 generation polyamidoamine (PAMAM) dendrimer with a dehydroabietylamine core, firstly was prepared through Michael addition and amidation, then the rosin-based dispersant was synthesized by the reaction of the dendrimer and chloroactic acid. The formulation and static stability of the slurry with the dispersant were investigated. The experimental results show that the rosin-based dispersant has a good dispersing ability at low dosage, and that the CWS with the dispersant exhibits the excellent static stability. Based on the above, the rosin-based dispersant is a promising dispersant for highly-loaded CWS in the practical use in industry.
\end{abstract}

\section{Intorduction}

This paper is concerned with synthesis and application of a new type of rosin-based dispersant for coal-water slurry (CWS) prepared from Chinese Shenhua coal. CWS, also referred to as coal-water fuel [1,2], has been regarded as a promising fuel instead of petroleum due to the rapid depletion of the latter [3], and many studies on CWS have been carried out in some countries such as China, Japan, America and Australia. A practical CWS as a liquid fuel should have such properties as an appropriate yield value to maintain its stability during storage, a low apparent viscosity for pumping and atomizing, and a high solid content for economical consideration.

In CWS technology, a dispersant plays a very key role. Many types of the dispersants for CWS have been reported so far, such as naphthalene sulfonates, copolymers of acrylic acid and other acrylic monomers, polyolefin series, lignosulfonates [4], humic acid salts and sulfonated humic acid salts, carboxylic acid salts and phosphate salts, non-ionic dispersing agents, etc.. However, most of the reported additives, synthesized by petroleum, are neither innocuous to the ecosystem nor to the environment. With increasing awareness of environmental protection, the research of natural renewable resources has attracted great attention.

Aiming at the existing status, in this study, rosin is used as raw material to prepare the dispersant for CWS, and the modifying reaction increases the hydrophilic property of rosin. The chemical reactions change the molecular structure of rosin thoroughly, and a new type of high-performance rosin series dispersant of CWS can be obtained. To estimate the performances of the synthesized dispersant, in the presence of the dispersant, The CWS from Chinese Shenhua coal was prepared, and the formulation and stability of the slurry with the dispersant were investigated.

\section{Experimental}

Materials. The preparation of CWS was carried out with Chinese Shenhua coal. Table 1 shows the results of the proximate and ultimate analyses of the coal sample.

Before the CWS preparation, the coal sample was dry-pulverized in a ball mill to obtain coal powder with the different particle size distribution by controlling the grinding time. The coal powder was screened through the variable-mesh sieves, and the particle size distribution of the coal sample 
following as: $8 \%$ for $20-40$ mesh, $42 \%$ for $40-120$ mesh, $7 \%$ for $120-200$ mesh, $8 \%$ for $200-300$ mesh, and 35\% for below 300 mesh.

Synthesis of 0.5-generation polyamidoamine dendrimer. $20 \mathrm{~g}$ dehydroabietylamine and $70 \mathrm{~g}$ methanol were mixed in a reactor flask fitted with a reflux condenser. Under stirring, the temperature was raised to $25{ }^{\circ} \mathrm{C}$ to render the reaction mixture dissolved. Then $50 \mathrm{~g}$ methyl acrylate was fed into the reactor by means of a dropping funnel. After the end of drop, Michael addition reaction is carried out at the temperature for $24 \mathrm{~h}$. Finally, the reaction mixture was distilled under reduced pressure, and the pale yellow viscous liquid, 0.5-generation polyamidoamine (PAMAM) dendrimer with a dehydroabietylamine core was obtained.

Table 1 Proximate and ultimate analyses of Chinese Bingchang coal

\begin{tabular}{|c|c|c|c|c|c|c|c|c|}
\hline \multirow[b]{2}{*}{ Component } & \multicolumn{3}{|c|}{ Proximate analyses/(wt\%, ad) } & \multicolumn{5}{|c|}{ Ultimate analyses/(wt\%, ad) } \\
\hline & $\mathrm{M}$ & $\mathrm{A}$ & $\mathrm{V}$ & C & $\mathrm{H}$ & $\mathrm{O}$ & $\mathrm{N}$ & $S$ \\
\hline Content & 7.68 & 4.59 & 33.01 & 82.22 & 4.69 & 11.59 & 0.91 & 0.26 \\
\hline
\end{tabular}

Synthesis of 1.0-generation polyamidoamine dendrimer. $20 \mathrm{~g}$ 0.5-generation PAMAM and 70 $\mathrm{g}$ methanol were fed into a reactor flask fitted with a reflux condenser. Under stirring, the temperature was raised to $25{ }^{\circ} \mathrm{C}$, and 20 g ethylenediamine was added into the reactor by means of a dropping funnel. After the end of drop, the amidation reaction is performed at the temperature for $24 \mathrm{~h}$. Finally, the reaction mixture was distilled under reduced pressure, and the yellow waxy solid, 1.0-generation polyamidoamine (PAMAM) dendrimer was obtained.

Synthesis of rosin-based dispersant. $10 \mathrm{~g}$ chloroactic acid and $20 \mathrm{~g}$ absolute ethanol were mixed in a reactor flask fitted with a reflux condenser, and the mixture was made with the $\mathrm{pH}$ of about 8 using aqueous sodium hydroxide of $40 \mathrm{wt} \%$. Under stirring, the temperature was raised to $60{ }^{\circ} \mathrm{C}$, and the mixture of $10 \mathrm{~g}$ 1.0-generation PAMAM and absolute ethanol was added dropwise into reactor. In the course of the drop, the $\mathrm{pH}$ of the reaction mixture was always kept around 8 with aqueous sodium hydroxide. After finishing the drop, The reaction mixture was maintained for $18 \mathrm{~h}$ till the end of the reaction. After cooled, the reaction mixture was recrystallized 3 times with absolute ethanol, and the pale yellow solid, the rosin-based dispersant was obtained.

Preparation of CWS. The coal powder was mixed slowly in a pot containing a known quantity of dispersant and deionized water. The contents were continuously stirred using a mixer during the addition of coal, and then the stirring was continued at $800 \mathrm{rpm}$ for another $10 \mathrm{~min}$ to ensure the homogenization of CWS. The slurry prepared was reserved for study of its characteristics.

Infrared spectrum analyses. A Fourier transform infrared spectrophotometer (VECTOR-22, Brucker Co., Germany ) was used for infrared spectrum analyses. The samples was dried under vacuum and mixed with potassium bromide in a mortar box, and the ground powder was pressed to discs for subsequent analyses. Infrared spectra were collected within a scanning range of 4000-400 $\mathrm{cm}^{-1}$.

Viscosity measurements. The viscosity measurements were performed employing a rheometer (AR-2000, TA Instrument Corp., USA). The CWS was stirred for 5 min before each measurement. The measurements were made at a shear rate of $100 \mathrm{~s}^{-1}$, and the temperature was kept within $25 \pm 2{ }^{\circ} \mathrm{C}$. The measured viscosity value is the apparent viscosity.

Zeta potential measurements. A zetasizer (ZEN3690, Malvern Instruments Corp., USA) was used for zeta potential measurements of the coal particles in the CWS. The CWS with different dispersant concentration was laid for $24 \mathrm{~h}$. After the slurry was centrifuged , the supernatant solution was diluted using distilled water to the suitable concentration. The diluted solution was stirred again by a stirrer for $5 \mathrm{~min}$. The mean value of zeta potential, which was measured three times at neutral $\mathrm{pH}$, is adopted in this study.

CWS stability experiments. The CWS prepared was transferred into a glass cylinder $(3 \mathrm{~cm}$ in diameter; CWS layer $15 \mathrm{~cm}$ in height) at room temperature for a definite period. Then the amount of water separated out from the slurry was measured. The staticstability of CWS was evaluated by the bleeding ratio, and the bleeding ratio was calculated as follows:

Bleeding ratio $=\mathrm{V}_{\mathrm{W}} / \mathrm{V}_{\mathrm{S}} \times 100 \%$

Where $\mathrm{V}_{\mathrm{W}}$ is the volume of bleeded water from CWS, and $\mathrm{V}_{\mathrm{S}}$ is the total volume of CWS. 


\section{Results and Discussion}

IR analyses. Fig. 1 shows the infrared spectra of (a) dehydroabietylamine, (b) 0.5-generation PAMAM dendrimer, (c) 1.0-generation PAMAM dendrimer and (d) the rosin-based dispersant. For 0.5-generation PAMAM dendrimer, the bands at 1736, 1194 and $1169 \mathrm{~cm}^{-1}$ are characteristic absorbance of ester group, which suggests the success of Michael addition reaction. In Fig. 1c, the band at $3288 \mathrm{~cm}^{-1}$ is assigned to N-H stretching vibration, and the bands at 1651 and $1556 \mathrm{~cm}^{-1}$ are related to amide groups. For the rosin-based dispersant, the bands at $1734 \mathrm{~cm}^{-1}$ is attributed to $\mathrm{C}=\mathrm{O}$ stretching vibration, and $1406 \mathrm{~cm}^{-1}$ is due to C-O stretching vibration, which proves the existence of - $\mathrm{COOH}$. Besides, the bands at $1640 \mathrm{~cm}^{-1}$ is attributed to amide groups. All of the above results demonstrate that the copolymer has the due structure.

Relationship between apparent viscosity and coal content. Relationship between apparent viscosity and coal content is reported in Fig. 2 (with a dispersant concentration of $0.4 \mathrm{wt} \%$, at a shear rate of $100 \mathrm{~s}^{-1}$ ). The apparent viscosity is found to increase with an increase of coal content. Beyond $68 \mathrm{wt} \%$ of coal content, the apparent viscosity can not be measured for the formation of a hard sediment of coal.

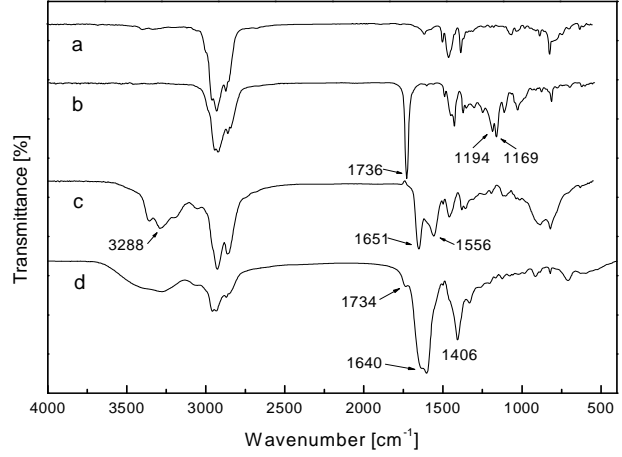

Fig. 1. Infrared spectra analyses.

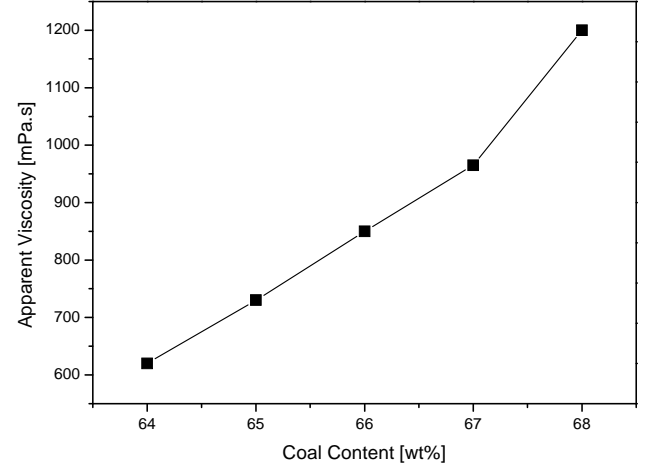

Fig. 2. Efect of coal content on apparent viscosity.

Effect of dispersant concentration on apparent viscosity and zeta potential. Apparent viscosity and zeta potential measurements are reported in Fig. 3 (with a coal content of $65 \mathrm{wt} \%$, at a shear rate of $100 \mathrm{~s}^{-1}$ ). With an increase of dispersant concentration, apparent viscosity and negative zeta potential decrease sharply when the dispersant concentration is less than $0.4 \mathrm{wt} \%$, and increase slightly when exceeds $0.4 \mathrm{wt} \%$. This tendency may be attributed to the multi-layer adsorption of dispersant molecules on coal surface, and many anionic dispersants for CWS have this characteristic.

The adsorbance amount of dispersant influences zeta potential. The adsorbance amount increases with an increase of dispersant concentration before adsorbance saturation [5], which results in the sharp decrease of negative zeta potential on coal surface. However, after the saturation point, excessive dispersant molecules arrange on the surface of compact adsorption layer in the opposite direction, resulting in the counteraction of surface electronegativity. Zeta potential, indicating coal interparticle electrostatic repulsion, and thereby, directly affects apparent viscosity.

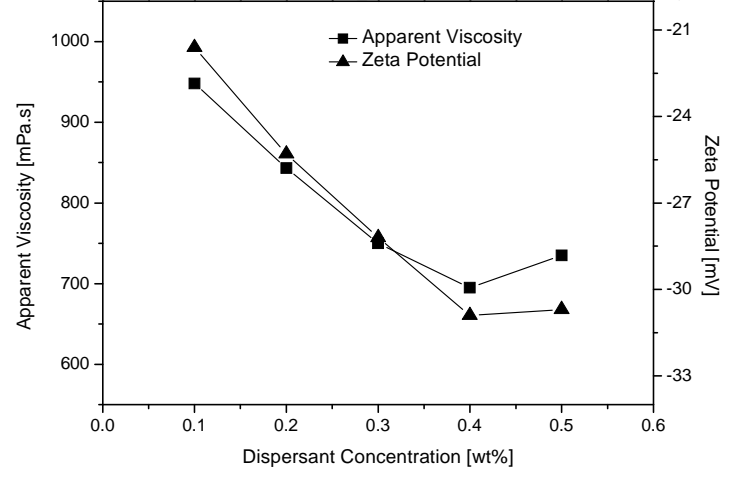

Fig. 3. Apparent viscosity and zeta potential curves.

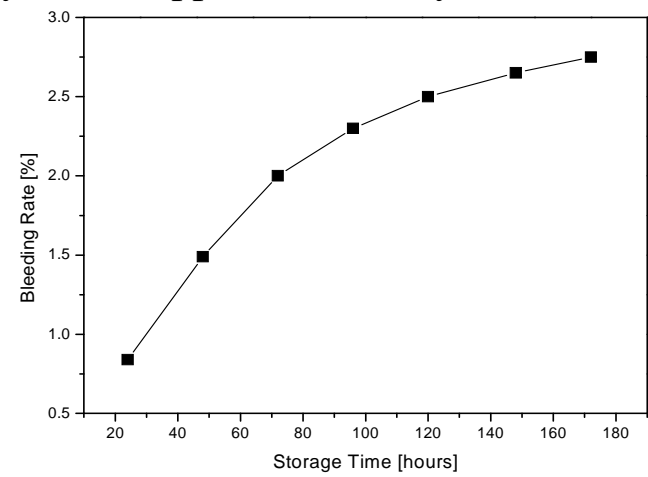

Fig. 4. Effect of storage time on bleeding rate of CWS.

CWS stability investigations. The stability measurements of the CWS (with a coal content of 65 wt $\%$ and a dispersant concentration of $0.4 \mathrm{wt} \%$.) taken at neutral $\mathrm{pH}$ are reported in Fig. 4 . The 
bleeding rate is found to rise with an increase of storage time, and however, as a whole, the bleeding rate of the slurry with the rosin-based dispersant keeps at a low level within the first $48 \mathrm{~h}$.

Electrostatic repulsion and steric wettability are the main factors influencing the stability of CWS $[6,7]$. When dispersant molecule is adsorbed on coal particle surface, The plenty of carboxyl groups introducted in the dispersant molecule cause stronger electronegativity of coal parpticle surface. The amide and amine groups in the dispersant molecule can form hydrogen band with water molecules, and increase the steric wettability of coal surface to eliminate the hydrophobic interaction. All of these have resulted in the excellent stability of the CWS.

\section{Summary}

A new type of rosin-based dispersant was synthesized through Michael addition, amidation and substitution reactions using dehydroabietylamine as raw material. The dispersant was used in the preparation of the CWS from Chinese Shenhua coal, and the formulation and stability of the slurry were examined. The experimental results show, at coal content of $65 \mathrm{wt} \%$, dispersant concentration of $0.4 \mathrm{wt} \%$ and shear rate of $100 \mathrm{~s}^{-1}$, the CWS gives the minimum viscosity of $695 \mathrm{mPa} \cdot \mathrm{s}$. The CWS with the rosin-based dispersant has excellent static stability. The excellent performances of the rosin-based dispersant attribute to it's peculiar structure. Furthermore, the rosin-based dispersant is considered to have a bright prospect owing to cheap cost and acceptable environmental pollution.

\section{Acknowledgements}

The authors would like to acknowledge the financial supports from National Natural Science Foundation of China (No.21176148) and Scientific Subject Foundation of Education Department of Shaanxi Province (No. 11JK0562).

\section{References}

[1] G. Papachristodoulou and O. Trass, Coal slurry fuel technology, The Can. J. Chem. Eng.. 65 (1987) 177-201.

[2] M. Pawlik, Polymeric dispersants for coal-water slurries, Colloids Surf. A: Physicochem. Eng.. 266 (2005) 82-90.

[3] D. Bienstock and E. M. Jamgochian, Coal-oil mixture technology in the US, Fuel. 60 (1981) 851-864.

[4] M. S. Zhou, X. Q. Qiu, D. J. Yang, H. M. Lou and X. P. Ouyang, High-performance dispersant of coal-water slurry synthesized from wheat straw alkali lignin, Fuel Process. Technol.. 88 (2007) 375-382.

[5] D. Das, S. Panigrahi, P.K. Senapati and P.K. Misra, Effect of organized assemblies. Part 5: Study on the rheology and stabilization of a concentrated coal-water slurry using saponin of the acacia concinna plant, Energy Fules. 23 (2009) 3217-3226.

[6] S. Aliferova, R. Titkov, V. Noveselov and N. Panteleeva, Application of nonionic surface-active substances in combination with acrylamide flocculants for silicate and carbonate mineral flotation, Miner. Eng.. 18 (2005) 1020-1023.

[7] P. Somasundaran, P. Chander and K. Chari, A study of the interaction between particles and bubbles in surfactants solutions, Colloids Surf.. 8 (1983) 121-136. 